\title{
Immune-Endocrine System Inspired Hierarchical Coevolutionary Multiobjective Optimization Algorithm for IoT Service
}

\author{
Zhen Yang, Yongsheng Ding, Senior Member, IEEE, Yaochu Jin, Fellow, IEEE, and Kuangrong Hao, \\ Member, IEEE
}

\begin{abstract}
The intelligent devices in Internet of Things (IoT) not only provide services, but also consider how to allocate heterogeneous resources and reduce resources consumption and service time as far as possible. This issue becomes crucial in the case of large-scale IoT environments. In order for the IoT service system to respond to multiple requests simultaneously and provide Pareto optimal decisions, we propose an immuneendocrine system inspired hierarchical coevolutionary multiobjective optimization algorithm (IE-HCMOA) in this paper. In IE-HCMOA, a multiobjective immune algorithm based on global ranking with vaccine (GRVIA) is designed to choose superior antibodies. Meanwhile, we adopt clustering in top population to make the operations more directional and purposeful and realize self-adaptive searching. And we use human forgetting memory mechanism to design two levels memory storage for the choice problem of solutions to achieve promising performance. In order to validate the practicability and effectiveness of IE-HCMOA, we apply it to the field of agricultural IoT service. The simulation results demonstrate that the proposed algorithm can obtain the best Pareto, the strongest exploration ability and excellent performance than NNIA and NSGA-II.
\end{abstract}

Index Terms-coevolutionary optimization, multiobjective optimization, immune-endocrine systems, hierarchical multipopulation, Internet of Things (IoT), services selection

\section{INTRODUCTION}

I nternet of Things (IoT) has quietly entered a multiple intelligence industry. Today in advocating energy saving and environmental protection, the configuration optimization of IoT resources has become an urgent problem to be solved.

This work was supported in part by the National Key Research and Development Plan from Ministry of Science and Technology (2016YFB0302701), in part by the National Natural Science Foundation of China under Grant 61473077 and Grant 61473078, in part by the International Collaborative Project of the Shanghai Committee of Science and Technology under Grant 16510711100, in part by Shanghai Science and Technology Promotion Project from Shanghai Municipal Agriculture Commission under Grant 2016-1-5-12, and in part by the Program for Changjiang Scholars from the Ministry of Education (2015-2019).

All the authors are with the Engineering Research Center of Digitized Textile and Apparel Technology, Ministry of Education, and also with the College of Information Science and Technology, Donghua University, Shanghai 201620, China (Corresponding author: Yongsheng Ding. e-mail: ysding@dhu.edu.cn). Yaochu Jin is also with the Department of Computer Science, University of Surrey, Guildford, Surrey GU2 7XH, United Kingdom. Zhen Yang is also with the College of Information Engineering, Huzhou University, Huzhou 313000, China.
There are many artificial intelligence algorithms for resource scheduling [1,2], which, however, were developed for specific applications and not well suited for solving IoT service problems studied in this work. IoT as a service [3], we will regard its entire layout as a service system. The solution to the resources optimization allocation problem of service-oriented networked collaborative equipment is a very complex issue, which belongs to a typical NP-hard combinatorial optimization problem [4]. How to minimize the consumption of resources, and shorten the service time? In other words, how to select multiple optimal services in enormous candidate sets to meet the above objectives? So it is going to be a challenging multiobjective optimization problem.

Many researchers have attempted to solve the problems of multiobjective service selection in Web service. Trummer et al. [5] presented the first approximation scheme for multiobjective quality-driven service selection. Chen et al. [6] launched research on multiobjective optimization of quality of service (QoS). They introduced Pareto set model for QoS-aware service composition. Wagner et al. [7] proposed an approach that supported decision makers in finding robust, QoS optimized service compositions using clustering. In our previous research work [8-10], we implemented adaptive web service composition inspired by the neuroendocrine-immune system. However, the above work focused on Web service composition based on QoS.

IoT services, whose features are large-scale, heterogeneity, unreliability and dynamic nature, are different from Web service. An important challenge to be addressed in the domain of IoT services composition is the development of efficient services selection algorithms for an optimal management of both energy and QoS [11]. This issue becomes crucial in the case of large-scale IoT environments composed of thousands of distributed entities. Jin et al. [12] stated that IoT is a paradigm in which real-world physical things can be connected to the Internet and provide services through the computing devices attached. A three-layer QoS scheduling model for service-oriented IoT was proposed by Li et al. [13]. The sensing as a service [14] model is expected to be built on top of the IoT infrastructure and services [15]. Then, Angelakis et al. [4] assigned services to interfaces with heterogeneous resources and produced optimal solutions for this 
computationally hard problem.

From the analysis of the above literatures, other approaches dealing with services selection were mostly unaware of energy issues, or they noted the minimum energy consumption but only as a unilateral goal. Study on multiobjective optimization in the context of IoT service is very few, especially in considering equipment energy consumption and service time. It is necessary to develop a multiobjective optimization algorithm for IoT service, which can offer a more practical value, e.g. intelligentized facility agriculture and industrial manufacture.

Evolutionary multiobjective optimization (EMO) has become one of the mainstream research directions in the field of evolutionary computation [16]. Zhou et al. [16] made a comprehensive review of the modern multiobjective evolutionary algorithms (MOEAs). Typical multiobjective optimization algorithms based on artificial immune systems (AIS) include multiobjective immune algorithm (MOIA) [17], constrained multiobjective immune algorithm (CMOIA) [18], an artificial immune network for multiobjective optimization (called vector immune system: VIS) [19], nondominated neighbor immune algorithm (NNIA) [20] and so on. Besides, in terms of vaccine, Jiao et al. [21] proposed the immune genetic algorithm (IGA) and gave strategies of selecting vaccines and constructing an immune operator. Woldemariam et al. [22] emulated a biological notion in vaccines to promote exploration in the search space. In recent years, a novel immune clonal algorithm (NICA) for multiobjective optimization was proposed in [23]. A degeneration recognizing clonal selection algorithm (DR-CSA) for multimodal optimization was designed in [24]. A new multiclass clustering method based on maximum margin clustering algorithm and immune evolutionary algorithm (IEMMMC) was proposed in [25]. In addition, some literatures [26-28] focused on local search strategies. In [28], a new multiobjective optimization framework based on nondominated sorting and local search (NSLS) was introduced. Li et al. [29] adopted a novel ranking strategy called global margin ranking (GMR) which deployed the position information of individuals in objective space to gain the margin of dominance throughout the population.

Furthermore, in order to consider the coordination between population and environment, population and population in the evolutionary process, coevolutionary mechanism has been introduced into the immune optimization algorithm, and good results have been obtained for solving combinatorial optimization problems [30]. The competition model and cooperative model are two important models in the coevolutionary multiobjective optimization algorithm (CMOA). The coevolutionary algorithm based on cooperative model has achieved great success in solving single objective optimization problem [31]. Tan et al. [32] presented a cooperative coevolutionary algorithm (CCEA) for multiobjective optimization, which was capable of maintaining archive diversity by dynamic sharing and extending operator. They also [33] proposed competitive-cooperation coevolutionary algorithm (COEA). Multiple subpopulations respectively optimized the part of decision variables. The difference is that the mapping relationship between each subpopulation and the decision variables are not fixed but determined by competitive results. The work [34] and [35] also employed coevolutionary technique and multiple populations for multiobjective optimization. Based on immune system model, several subpopulations evolved using different evolutionary strategies $[36,37]$. An immune coevolutionary algorithm with two stages was designed to search the optimal balanced partitions by $\mathrm{Hu}$ et al. [38]. They also [39] proposed a coevolutionary immune algorithm for garment matching problem, introducing dominance affinity and distance affinity. Shang et al. [40-42] proposed cooperative coevolutionary algorithms for multiobjective capacitated arc routing problem. Tian et al. [43] employed two subpopulations that were cooperatively coevolved using the coevolutionary algorithm to achieve a better global optimality for the estimated radial basis function neural network. Mu et al. [44] proposed a novel coevolutionary mechanism based on elite strategy, where elite individuals were used to guide the search. In [45], a framework named hyper multiobjective evolutionary algorithm (HMOEA) was proposed. The size of subpopulation was adjusted according to the corresponding MOEA's performance. Wang et al. [46] adopted multiple subpopulations, and used clustering and statistical methods to guide the generation of new population and the local search. Moreover, some researchers decomposed a multiobjective optimization problem in a collaborative manner [47-48]. In addition, inspired by the mammalian endocrine system, an artificial endocrine controller for power management is designed in robotic systems [49]. In [50], the endocrine mechanism was introduced to regulate cooperative coevolution among the particles.

The aforementioned research work usually adopts multipopulation to implement coevolution; nevertheless it is rare to organize the populations in a hierarchical way. Meanwhile, little has been done to embed the endocrine regulation mechanism into the evolution of subpopulations in multiobjective immune algorithm, while there is a natural synergy between the immune system and the endocrine system [51]. Thus, the main motivation of this work is to simulate the functions of immune-endocrine system and map these mechanisms to the coevolution of multiple populations, so as to more efficiently solve the multiobjective optimization of IoT service problems. Inspired by the existing achievements and the human immune-endocrine mechanism, we propose an immuneendocrine system inspired hierarchical coevolutionary multiobjective optimization algorithm (IE-HCMOA) in this paper. IE-HCMOA employs the hierarchical structure, i.e. foundation layer and top layer, which evolves and learns from the ideas similar to previous elite strategy but not the same. It can provide optimal nondominated decision-making for service-oriented resources optimization allocation problem in IoT systems. Experimental results demonstrate the proposed algorithm is efficient to minimize the consumption of resources and shorten the service time.

The main contributions of this paper are as follows: (1) Inspired by the endocrine regulation mechanism, an endocrine-based strategy is designed and embedded in the subpopulation evolution process, which can guide efficient 
cooperative interactions among subpopulations and assist the top population towards global optimal solutions. (2) The human forgetting memory mechanism is introduced into the evolution of the top population, which successfully solves the choice problem of nondominated solutions. (3) Using clustering and statistical method during the evolution process, difference components operated on $X$ axis and $Y$ axis are proposed, which can make the operations more directionally and purposefully.

The rest of this paper is organized as follows. Section II defines the multiobjective optimization model of IoT service. Section III proposes IE-HCMOA, and details search mechanisms and strategies. The performances of the proposed approaches are evaluated and discussed in Section IV. Section $\mathrm{V}$ concludes the whole paper.

\section{MultiobJective Optimization Model of IoT SERVICE}

\section{A. IoT Service Model}

IoT is a bridge connecting the physical world and the information world. The physical world has a large number of pervasively distributed and interconnected smart objects (devices, sensors, actuators, appliances, etc.). In this auto-organized or intelligent IoT environment, sensors will capture potentially enormous amounts of data, which is then sent to the information world. These data streams can be analyzed and denoted as service requests, and then smart devices offer them a multitude of appropriate quality services.

The question is how to select the best matching services for multiple requests to minimize the consumption of resources, and shorten the service time? As to the above problem, Fig. 1 describes the IoT service model based on the proposed algorithm in this paper. IoT service region is a served object with layout of the sensing devices, which belong to different kinds of sensors, coding them $A, B, C$, etc. There are many sensors in each kind of sensor, e.g. $A$, coding them $A 1, A 2, A 3$, etc. Monitored data converge into service requests streams and arrive at IoT service platform via the network layer. Firstly, the platform analyzes and categorizes the requests data by its type. Next it invokes IE-HCMOA which runs by three stages. (1) Search a variety of possible matching services schemes in enormous available candidate services sets. (2) Compute the total cost and service time of each scheme in each generation of evolution. (3) Solve nondominated solutions and obtain Pareto optimal services selection schemes. Then IoT service platform notifies the selected smart devices according to specific job requirements. It is worth noting that there are thousands of intelligent devices in a large server-side, which act as service providers. Finally, the selected devices provide services for this IoT service region. The whole process realizes the unsupervised intelligent management.

To describe clearly the IoT service model, a service request is expressed as a four-tuple, whose definition is represented as follows:

(Request_id, Type, Workload, Location).

where Request_id indicates the unique identification code of the service request. Type indicates the type of request, which is due to heterogeneous devices which produce various requests.
Workload depends on collected data from all kinds of sensors. Location represents geographical coordinates of the request.

When a service provider accepts the response of service request, it is expressed as a six-tuple, whose definition is shown as follows:

(Service_id, Type, u, e, $\delta$, Location).

where Service_id indicates the unique identification code of the service provider. Type denotes the type of service. $u$ indicates the using state of the service. $e$ represents the unit energy consumption. $\delta$ denotes the ability value. Location represents the geographical coordinates of the service.

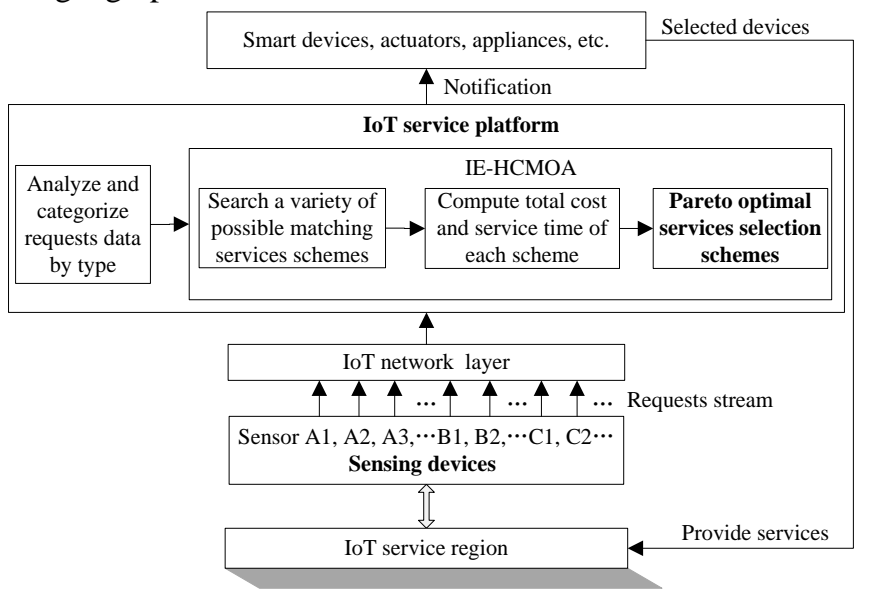

Fig. 1. IoT service model.

\section{B. Multiobjective Optimization Model}

In order to select service providers reasonably and assign the workload of the service requests, we design a multiobjective optimization model aiming to make the final energy consumption of system resources and service time minimum.

Assume that there are $N$ available services and $M$ requests per unit time; we obtain a bi-objective function $F$ as shown in Eq. (1), which is to minimize the total service cost and the service time.

$$
\text { minimize } F=(\text { Total cost, Service time })
$$
where,

$$
\begin{aligned}
& \left.f_{1}=\text { Total cost }=\sum_{i=1}^{N} \sum_{j=1}^{M} x_{i j}\left(\operatorname{dist}\left(s_{i}, r_{j}\right)+e c\left(s_{i}, r_{j}\right)\right)\right) \\
& f_{2}=\text { Service time }=\max _{i=1}^{N}\left\{S T_{i}\right\}
\end{aligned}
$$

In Eq. (2), $\operatorname{dist}\left(s_{i}, r_{j}\right)$ shows the transmission cost between service $s_{i}$ and request $r_{j}$, and we use Euclidean metric to calculate it, as shown in Eq. (4). ec( $\left.s_{i}, r_{j}\right)$ shows the cost of energy consumption between service $s_{i}$ and request $r_{j}$, as shown in Eq. (5).

$$
\begin{aligned}
& \operatorname{dist}\left(s_{i}, r_{j}\right)=\sqrt{\left(X_{i}-X_{j}\right)^{2}+\left(Y_{i}-Y_{j}\right)^{2}} \\
& e c\left(s_{i}, r_{j}\right)= \begin{cases}\text { workload }_{j} * p_{i} * e_{i} & , x_{i j}=1 \\
0 & , x_{i j}=0\end{cases}
\end{aligned}
$$

In Eq. (5), $p_{i}$ denotes the quotient of workload distributed to service $i$. workload $_{j}$ is the workload of request $j . e_{i}$ represents the unit energy consumption of service $i$. A service provider is only assigned to one request in a moment.

In Eq. (3), $S T_{i}$ of each service $i$ depends on the amount of work done and its own ability $\delta_{i}$, as shown in Eq. (6). The number of all the required services should be less than the total 
number of available services. If not, part of the requests must be waiting. $x_{i j}$ represents that whether service $i$ is assigned to request $j$. Corresponding constraints are given in Eq. (7) and Eq. (8).

$$
S T_{i}=\text { workload }_{i} / \delta_{i}
$$

s.t.

$$
\begin{aligned}
& \sum_{j=1}^{M} x_{i j}=1, i=1 \ldots N \\
& x_{i j} \in\{0,1\}, \forall i, j
\end{aligned}
$$

\section{HIERARCHICAL COEVOLUTIONARY IMMUNE-ENDOCRINE ALGORITHM}

In this section, we introduce the proposed IE-HCMOA for the multiobjective optimization of IoT service as shown in Fig. 2. IE-HCMOA is inspired by the existing mechanism of human immune-endocrine system.

There is a natural synergy between the immune system and the endocrine system [51]. When a harmful foreign matter (i.e. antigen) strikes an organism, a series of immune reactions occur in the immune system, which is the process of immune response. During this process, immune regulation makes immune response maintain an appropriate intensity to ensure the stability of the internal environment. It includes: (1) interactions among immune cells, (2) the regulation between immune system and other systems (e.g. nervous system and endocrine system), (3) positive regulation between immune complexes and antigen presenting cells, (4) the regulation of antibody affinity, etc.

The origin of the structure of IE-HCMOA draws on two aspects among them. One is the regulating effect of immune cells, which maps the hierarchical structure; the other is regulatory actions of the endocrine system, which maps the communication and collaborative work among subpopulations. Hormones secreted by the endocrine system play a prominent role in immune regulation. The endocrine system completes the regulation of immune enhancement and suppression through the transmission of activator hormones (HA) and inhibitor hormones (HI).

In addition, immune memory is another important feature of the immune system. When the body is exposed to certain antigens and then contacts the same antigen again, the latent period of antibody is significantly shorter than that of initial response. Meanwhile the immune system can produce stronger and higher affinity antibodies than the primary immunization. Using this characteristic, we can associate immune memory with the nondominated solutions of the subpopulation. Doing so can help the subpopulation to find better solutions than the previous generation.

In IE-HCMOA, each population corresponds to an aggregation group of antibodies of immune system. Vaccine boosts the immune system to beat an antigen. Prior knowledge (i.e. solutions from another algorithm) acts as vaccines. That is to say it can assist IE-HCMOA to seek out better solutions. The hierarchical structure is proposed to divide the function of each population. The top layer and the foundation layer establish the

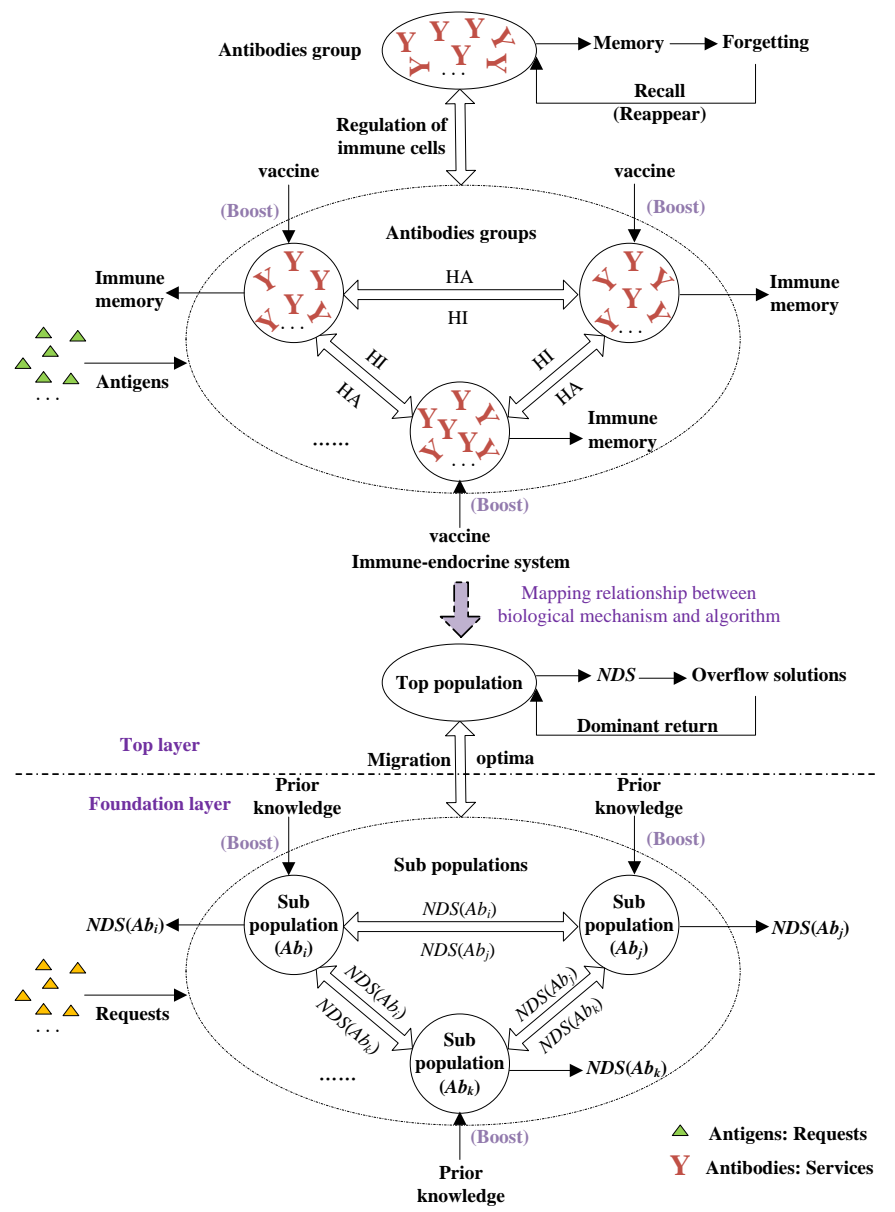

Fig. 2. Intelligent model of IE-HCMOA inspired by immune-endocrine system.

connection by migrating optima like immune cells. The top population situated at the top layer is mainly responsible for improving the accuracy of the solutions. The subpopulation situated at the foundation layer is responsible for exploring a larger solutions space and accelerating the convergence. They rely on hormones for communication among subpopulations, which cooperatively complete the work through the transmission of HA and HI. Nondominated solutions (NDS) of subpopulations respectively act as HA and HI. Subpopulations adopt a variable memory capacity. The top population uses the forgetting memory mechanism and builds a two-level memory storage mode. Due to its own characteristics, the top population employs a fixed memory capacity. Details will be presented in Section III (D).

In order to better understand, the flowchart of IE-HCMOA is shown in Fig. 3. In Fig. 3, NONPOP, DOMPOP, and NDS represent nondominated population, dominated population, and nondominated solutions, respectively. Specific steps of IE-HCMOA mainly involve of the following parts.

(1) Initialization. Produce initial antibodies and compose $m$ subpopulations.

(2) Evolution of subpopulations. Evolve subpopulations via a multiobjective immune algorithm based on global ranking with vaccine (GRVIA) which will be introduced in Section III(A) and obtain NDS and dominated solutions (DOMS) of each subpopulation.

(3) Hormone calculation. Calculate hormone of each 
subpopulation according to the subsequent Eq. (13) in Section III(B).

(4) Establishment of the top population. All the NONPOPS of subpopulations migrate to the top population. Its rest is filled by part of DOMPOPs according to the subsequent Eq. (15) in Section III(B).

(5) Evolution of the top population.

If the generation of evolution satisfies a certain frequency (every five generations), clustering is performed. According to two kinds of operators (Eq. (16) and Eq. (17) in Section III (C)), personalized crossover operation is accomplished. Then execute vaccine injection based on the results of the above step.

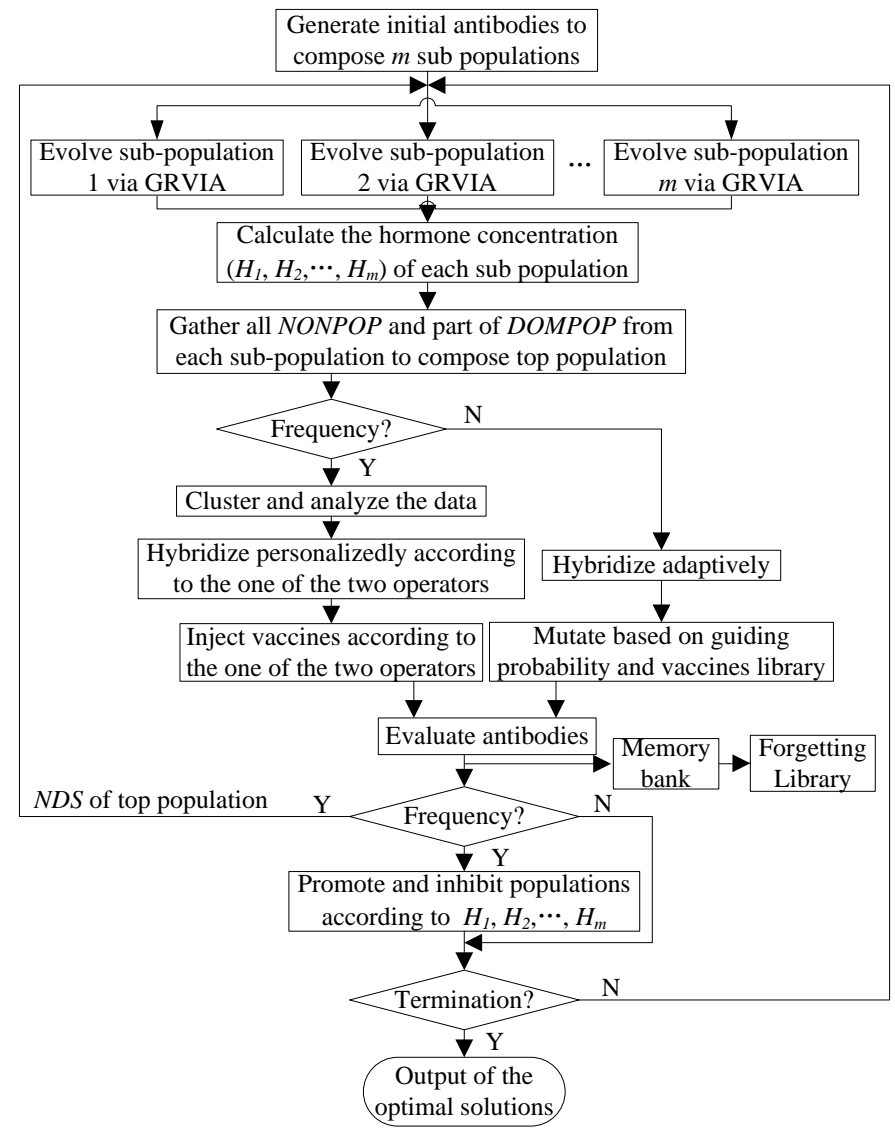

Fig. 3. Flowchart of IE-HCMOA.

If the generation of evolution does not satisfy a certain frequency, crossover operation is performed in terms of the adaptive probability $P_{c}$ in Eq. (10) of Section III(A). Next mutation operation is executed in accordance with equal probability to replace a gene. One of replacement sets is from the vaccines library; the other is from the service providers with lower energy consumption.

(6) Evaluation. It evaluates each antibody through fitness calculation and crowding distance comparison, and finds NDS of the top population.

(7) Updating memory and forgetting units.

(8) Cooperative interaction of populations. If the generation of evolution satisfies a certain frequency, information is exchanged among subpopulations based on hormones.

(9) Circulation. Turn to Step (2) until the termination condition is met.

\section{A. GRVIA}

In this section, we design GRVIA for multiobjective IoT service selection, whose effectiveness can provide the basic guarantee for subpopulation evolution of IE-HCMOA. The framework of GRVIA is explained in Fig. 4.

1) Crossover and correction operation

GRVIA adopts real number coding, which represents the chosen service device, and uses multipoint crossover strategy under a dynamic crossover probability $P_{c}$ to get a new antibody. For each antibody $a_{i}$, the first and last positions of multipoint crossover are two random numbers in the range of solution dimensions. The positions for performing multipoint crossover are generated using Eq. (9), where Dim denotes the dimension of the decision space, and rand is a random number between 0 and 1 .

$$
\text { Position }=\lceil\text { Dim } * \text { rand }\rceil
$$

Based on the antibody's fitness, an adaptive crossover equation is designed as in Eq. (10).

$$
P_{c}=\frac{1-e^{-2\left[\cos \left(\left(F_{\max }-F\left(a_{i}\right)\right) /\left(F_{\max }-F_{\min }\right)\right)\right]}}{1+e^{-2\left[\cos \left(\left(F_{\max }-F\left(a_{i}\right)\right) /\left(F_{\max }-F_{\min }\right)\right)\right]}}
$$

where $F_{\max }$ and $F_{\text {min }}$ are the maximum and minimum fitness values in all the antibodies, respectively; $F\left(a_{i}\right)$ denotes the fitness value of antibody $a_{i}$ under objective function $f_{1}$. This adaptive crossover probability $P_{\mathrm{c}}$ is more effective because it considers fitness value $F\left(a_{i}\right)$ of each antibody. $P_{\mathrm{c}}$ increases as the value of $F\left(a_{i}\right)$ increases, which makes antibody $a_{i}$ have more opportunities to cross with good antibodies.

As for correction operation, some genes are likely to be repeated in crossover and mutation process, hence they should be corrected so as to meet constraint (7). If there are repeated numbers between crossover bits and the rest bits, repeated numbers in the rest bits are replaced by the stochastic elements from the complementary set of $1 \sim N$ service providers and the current solution.

2) Immune selection operator

The computing method of immune selection operator is different from the single objective. Firstly, the function values of the bi-objective are taken into consideration in computing the fitness probability of antibody. Secondly, the crowding distance of each antibody is included in the calculation of immune selection operator. Most multiobjective optimization algorithms give priority to the individuals with the better convergence value. Distribution indicator is at the secondary position. However, we fully consider the two indicators and design immune selection operator based on global ranking (GR) as shown in Eq. (11). It is worth noting that GR here considers the affinity of each individual, crowding degree throughout the population and optimal weight assignment of the two parties aiming at the problem (1). The fitness of antibody is defined as the affinity between antibody $k$ and antigen. The formula is stated in (12).

$$
\begin{aligned}
& P_{\text {choose }}=w \cdot P_{\text {fitness }}+(1-w) \cdot P_{\text {crowding }} \\
& (A g)_{k}=\alpha(A g)_{k}^{f_{1}}+\beta(A g)_{k}^{f_{2}}
\end{aligned}
$$

where $P_{\text {fitness }}$ represents the ratio, i.e. the fitness of an antibody to the sum of fitness of the entire population. $P_{\text {crowding }}$ denotes the crowding degree probability of antibody. It is similar to the 
probability of antibody concentration. The calculation method is the same. The only difference is that when the crowding distance [20] is higher, $P_{\text {crowding }}$ and $P_{\text {choose }}$ will be bigger; when the crowding distance is lower, $P_{\text {crowding }}$ and $P_{\text {choose }}$ will be smaller. Accordingly, antibodies with large crowding distance are promoted; vice are suppressed. $w$ and 1- $w$ represent respectively the weight of fitness and crowding distance. $w$ is set as 0.7 (refer to discussion in Section IV(E)). $\alpha$ and $\beta$ represent the weight of the affinity under the objective function $f_{1}$ and $f_{2}$, respectively, and their values are set as 0.7 and 0.3 . The sum of $\alpha$ (refer to discussion in Section IV(E)) and $\beta$ is equal to 1 .

3) Vaccination

From a biological point of view, evolution is the process of population optimization through the selection mechanism of survival of the fittest. Immunity is a means for organisms to protect themselves through the neutralization reaction of antibody and antigen. It has memory characteristics and can identify populations and individuals. If we treat our evolutionary algorithms as an organism, the inevitable degeneration phenomenon in the algorithm can be regarded as foreign antigen. The algorithm uses feature information of the problem as vaccine and then suppresses the above degradation phenomenon by injecting "vaccine" [22]. It can be regarded as a neutralization reaction process. Therefore, vaccines are used to enhance immunity and effectively accelerate the convergence.

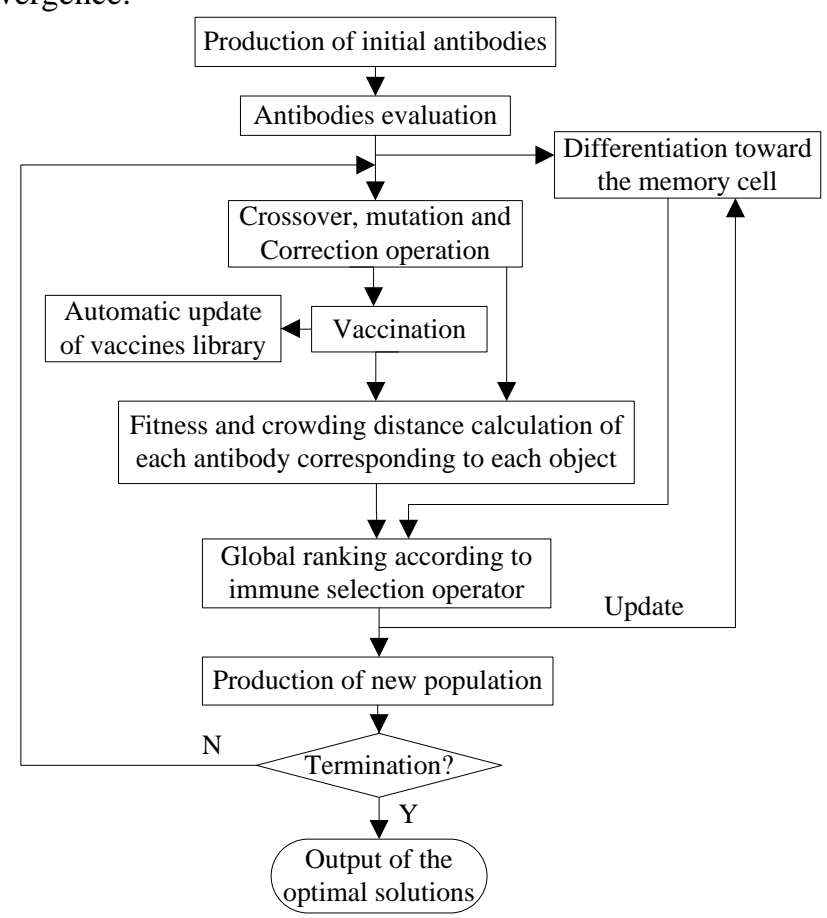

Fig. 4. Framework of GRVIA.

This paper uses NNIA [20] to test the above problem (1). By comparing and analyzing the results, we have found that there are obvious rules and characteristics of some gene positions. Some genes are the same numbers (i.e. service providers) in some optimal solutions; meanwhile they contain numbers which focus on a specific data set. In view of this, vaccines are extracted and then injected into GRVIA to enhance the exploration of global and local optima and achieve promising performance. Here it is necessary to note that the best vaccine individual is chosen as initial vaccine $_{\text {best }}$ and the vaccine library is at first built based on the data set from the optimal solutions of NNIA. The process of injecting vaccine is described in Algorithm 1. Vaccine is updated automatically in the process of GRVIA evolution, which is also drawn in Fig. 4. Another point to note is that random $30 \%$ individuals are injected vaccine.

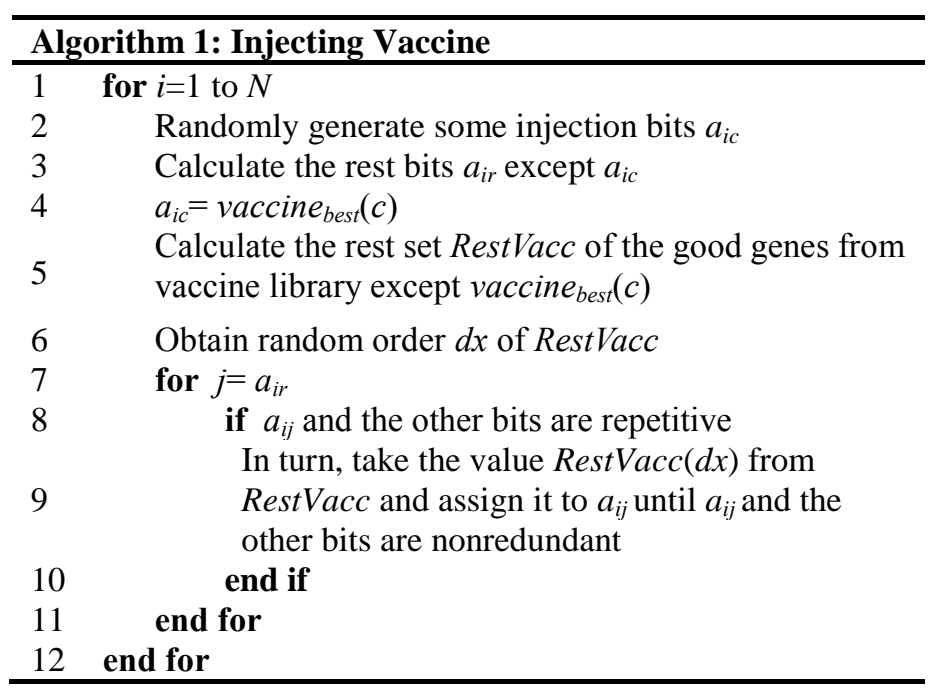

\section{B. Hormonal Regulation}

Multiple populations are regards as a whole biological system, which is through endocrine regulation among populations. Dividing the population into $m$ subpopulations can effectively maintain the diversity of individuals in the population. It can effectively avoid the immature convergence of the evolutionary computation with single population.

In IE-HCMOA, subpopulations evolve independently. When they evolve to a certain degree, i.e. satisfy a certain frequency, endocrine glands also secrete hormone to reach the amount of interaction. Hormone includes two types: activator hormones (HA) and inhibitor hormones (HI). HA and $\mathrm{HI}$ belong to hormone. HA enhances immune function and HI suppresses immune function. When the number of nondominated solutions of a subpopulation $A b_{j}$ is the most, this represents that the level of secreted HA of $A b_{j}$ is the highest. Conversely, when the number of nondominated solutions of a subpopulation $A b_{k}$ is the fewest, the level of secreted $\mathrm{HI}$ of $A b_{k}$ is the lowest. At this time let the two sub groups $A b_{j}$ and $A b_{k}$ to communicate together and exchange excellent individuals, which can expand the search range of the entire population and is conducive to jump out of local optimal value for the algorithm. The goal is to make the whole biological system develop towards a global equilibrium state. Subpopulations make progress together by means of cooperation to complete the evolutionary task ultimately. Using hormonal regulation mechanism of biological endocrine system and frequency control, IE-HCMOA can keep the best combination of convergence speed and population diversity, ultimately receive good results. Here frequency control means that hormone regulation has a time interval, e.g. every five generations.

The calculation formula of hormone concentration is given 
in Eq. (13).

$$
H\left(A b_{j}\right)=\frac{l_{j}}{\sum_{j=1}^{n} l_{j}}
$$

where $l_{j}$ is set according to the Eq.(14), which specifies the number of $N D S$ in subpopulation $A b_{j} . n$ indicates the number of subpopulations.

$$
l_{j}=\operatorname{Num}\left(N D S\left(A b_{j}\right)\right)
$$

Number of $D O M P O P$ entering into top population depends on $H\left(A b_{j}\right) . N M$ is the size of top population. memorySize is the current size of the memory bank. It can be set as follows.

Num_DOMPOP $\left(A b_{j}\right)$

$$
=\left(N M-\sum_{j=1}^{n} l_{j}-\text { memorySize }\right) \cdot H\left(A b_{j}\right)
$$

\section{Clustering}

The clustering is to calculate operation probability, which makes the subsequent operations more directional and purposeful. This operation probability is determined by the gap between antibodies and clustering centers (i.e. the nondominated solutions). The clustering centers here are different from the central points of public understanding, which are not the centers of the geographical location but nondominated solutions closest to axes, as shown in Fig. 5. Solid circles mark for $N D S$ and denote clustering centers.

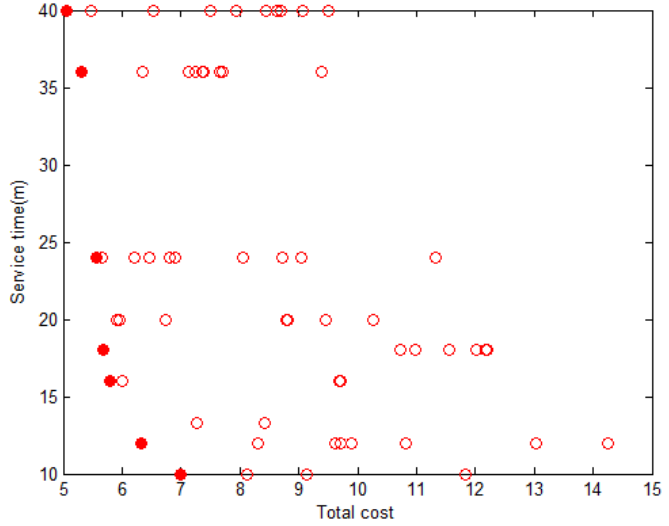

Fig. 5. Display of the candidate service sets in a generation.

Antibodies belonging to the same cluster set are classified into two categories, which are clustering center points $C C$ and complementary set $C S$. Antibodies in the $C C$ and $C S$ follow different operators. The former is associated with the number of iterations, which decreases with the increase of the number of iterations. It is updated according to Eq. (16). The latter determines the difference with this kind of the clustering center points. It consists of different components at the direction of the $X$ and $Y$ axis, whose calculation equation is set according to Eq. (17). For antibody $a_{i}$ in the $C S$, operators at the direction of the $X$ and $Y$ axis follow different Eqs. (18) and (19). The more different the values of the objective functions are, the more significant the approaching effect is. In other words, the greater the probability of operation is.

$$
\begin{aligned}
& P_{\text {opeator }}^{c c}=p_{\max }-\left(p_{\max }-p_{\min }\right) \cdot t / T \\
& P_{\text {opeator }}^{c s}=X_{o p}+Y_{o p} \\
& X_{o p}^{a_{i}}=\frac{F\left(a_{i}\right)^{f_{1}}-F_{\min } X^{f_{1}}}{F_{\max } X^{f_{1}-F_{\min }} X^{f_{1}}} \\
& Y_{o p}^{a_{i}}=\frac{F\left(a_{i}\right)^{f_{2}}-F_{\min } Y^{f_{2}}}{F_{\max } Y^{f_{2}}-F_{\min } Y^{f_{2}}} \cdot 0.1
\end{aligned}
$$

From the clustering characteristics of this problem, the points in the $C S$ are very close to those in the $C C$ on the $Y$ axis component. In most cases, $Y_{o p}$ equals to one. However, to adjust dimensional consistency of $Y_{o p}$ and $X_{o p}$ and we expect operating on the $X$ axis direction is dominant, thus $Y_{o p}$ is multiplied by a scaling factor 0.1 , as indicated by Eq. (19). When $X_{o p}$ or $Y_{o p}$ is equal to zero, it is uniformly reassigned to 0.02 during the evolutionary process. The reason to do so is that we expect the evolution operations to occur at a smaller probability at the direction of that equal to zero. Superscript $f_{1}$ and $f_{2}$ indicate fitness calculation under objective function $f_{1}$ and $f_{2}$.

Cluster analysis is applied when the populations evolve to certain degree, then a number of cluster areas can be obtained, where the cluster centers are located nearby the corresponding Pareto front (PF). If clustering is too often, it not only increases the amount of computation, but also may not receive the desired results because the solutions have not yet evolved to maturity.

The clustering algorithm is presented in Algorithm 2. Let $|N O N P O P|$ and $|D O M P O P|$ be the current size of NONPOP and $D O M P O P . n_{\text {cluster }}$ is the threshold for performing clustering which is set as 3 . When $|N O N P O P|$ is equal to 1 or 2 , the diversity of $N D S$ is too little. In such a situation, clustering does not make sense and costs computation time.

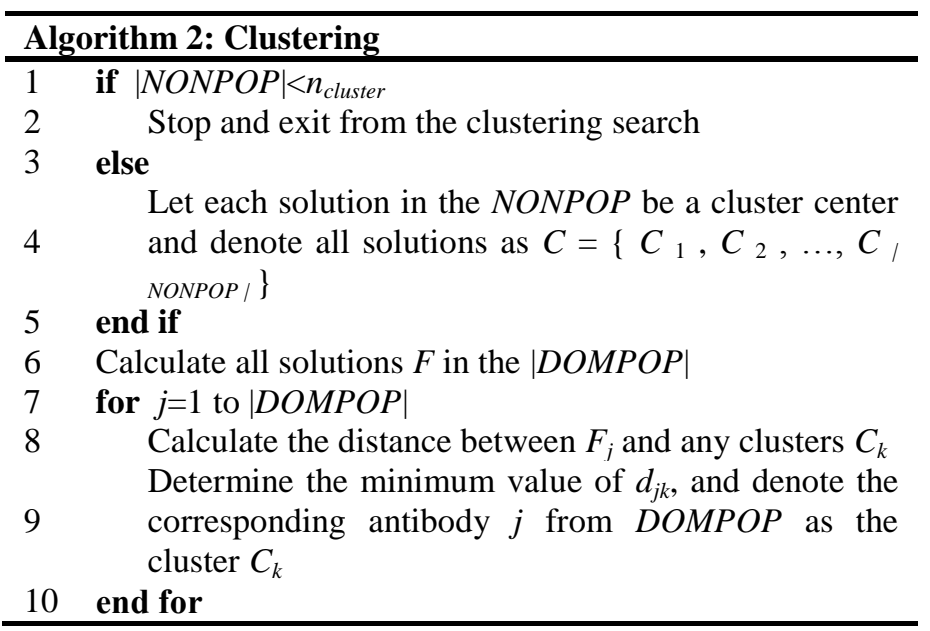

\section{Forgetting Memory Mechanism}

Most algorithms consider memory performance and few consider forgetting characteristics. Ebbinghaus had discovered forgetting is regular [52]. The process of forgetting is first very fast; after that it is gradually slow down. He had confirmed traces of human memory will fade over time. Finally it cannot be retrieved or identified so that our memories cannot be reproduced. The human forgetting memory mechanism was successfully introduced into the algorithms [53-55].

There are two motivations for using the forgetting memory mechanism. Firstly, as is known to all, human memory is fading as time passes, but something that has been forgotten may be recalled when similar scenario appears again. Secondly, the antibodies remain in the body for about three weeks. After that, they will disappear in order to make room for the new antibodies.

Since the top population consists of some antibodies of several subpopulations in IE-HCMOA, updating the top 
population is regularly needed during evolution. In order to make the algorithm more robust, we only let high-level memory bear this responsibility. And a certain amount of fixed memory capacity is needed to maintain the guiding force. However, aiming at the multiobjective optimization problem, the choice of solutions within the memory capacity is also a problem. In view of this, using human forgetting memory mechanism, two levels memory storage mechanism is designed for the choice problem of solutions to achieve promising performance.

Specific algorithm of the forgetting memory mechanism is described in Algorithm 3.

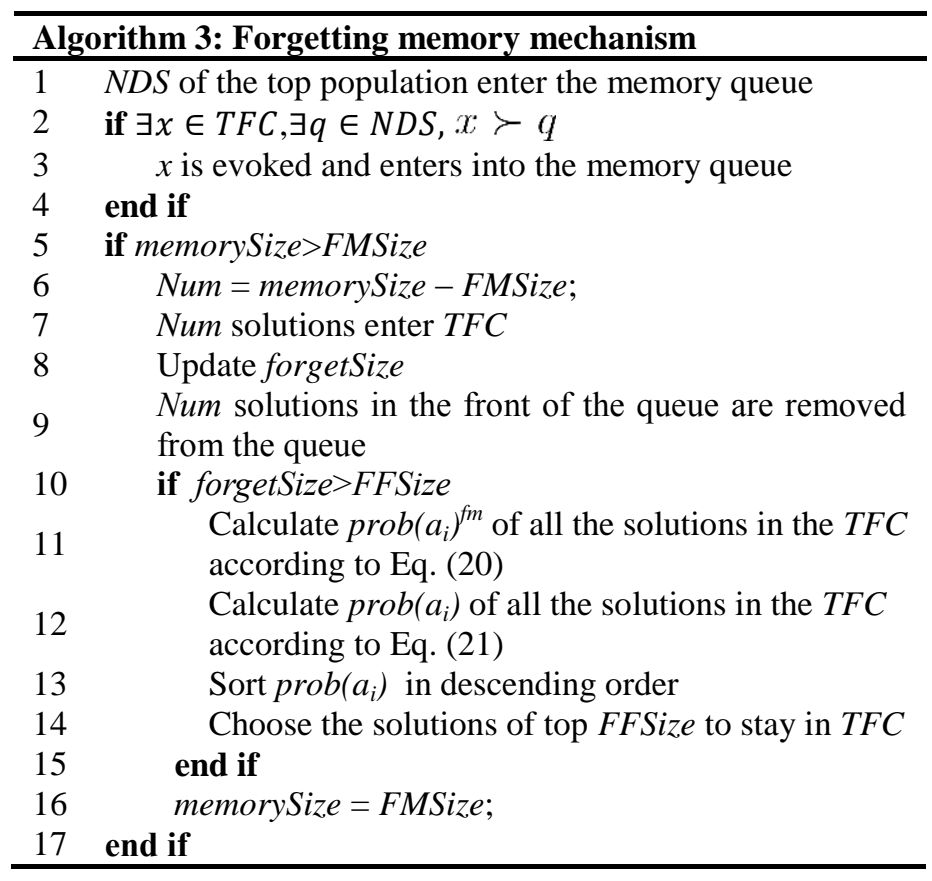

In Algorithm 3, FMSize denotes fixed memory capacity. FFSize indicates fixed temporary forgetting capacity (TFC). Let memorySize and forgetSize be the current size. When the contents of the memory bank overflow, the overflowed memories are moved into TFC. The first level of memory adopts the queue storage mode following the principle of first-in-first-out (FIFO). Or it could be called rolling memory. The second level of memory, i.e. temporary forgetting unit, adopts competitive probability model to evaluate either a stay or a permanent forgotten. The formulas of competitive probability model are as shown in Eq. (20) and Eq. (21).

$$
\begin{aligned}
& \operatorname{prob}\left(a_{i}\right)^{f_{m}}=\frac{i n d e x\left(a_{i}\right)^{f_{m}}}{\operatorname{forgetSize}}, m=1,2 . \\
& \operatorname{prob}\left(a_{i}\right)=\max \left\{\operatorname{prob}\left(a_{i}\right)^{f_{m}}\right\}, m=1,2 .
\end{aligned}
$$

All the solutions in the TFC are sorted according to the values of the objective function $f_{m}$ in descending order respectively, and their corresponding index values are obtained. $\operatorname{prob}\left(a_{i}\right)^{f m}$ of the antibody $a_{i}$ is calculated according to Eq. (20). Then, by comparing two probabilities $\operatorname{prob}\left(a_{i}\right)^{\text {fm }}$ of $a_{i}$, the maximum value is taken as the final competitive probability of $a_{i}$. Finally, the choice of solutions is implemented from lines 13 to 14 in Algorithm 3.

\section{Performance Evaluation with ApPlication to AGRICULTURAL IOT SERVICE}

In order to validate the practicability and effectiveness of IE-HCMOA, it is applied to the field of agricultural IoT service scenarios.

For the agriculture greenhouse control system based on IoT, all kinds of sensors are set for monitoring the growth of all kinds of vegetables and fruits. There are temperature sensors, humidity sensors, soil moisture sensors, nutrient elements sensors, and carbon dioxide sensors, which are used to measure environment of temperature, relative humidity, soil water content, soil nutrient, carbon dioxide concentration and other physical parameters. Monitored data are gathered, analyzed and processed. We regard the processed data which meets certain conditions as a service request. In this agricultural IoT scenario, there are some equipment, such as irrigation machine, fertilizer applicator and pesticide spraying machine. We regard them as a service provider. The question to be solved is that the algorithm designed should be able to select the best matching services for multiple requests to make the total cost and service time minimal.

Agricultural IoT is different from common network, which is subject to geographical restrictions. For example, the arm length of the service device is limited. If the obtained solution (service provider) is beyond its service range, this service scheme is meaningless. Thus, based on the features of deployment costs and service practices in actual agricultural IoT, the following simulation scale must be suitable, and all the matching services are within their service ranges.

\section{A. Experimental Setting}

In order to test the performance of the algorithm, we conduct two test cases respectively. We call them as Case One and Case Two. In Case One, an agricultural IoT area $(160 \times 160)$ is designated. The number of sensor nodes (i.e. service requests) is set to 200 so as to ensure sufficient requests. In addition, the number of agricultural IoT device nodes (i.e. service providers) is set to 200. The coordinates of requests and services are randomly generated. The number of service requests is uncertain in the context of the actual agricultural IoT scenarios. In this test case, we assume that the number of random service requests belonging to the same type is 20 and service facilities are all available at that moment in the current agricultural IoT service system. That is to say the system will respond to these 20 requests and select 20 optimal service providers to complete corresponding tasks of watering or fertilizing. From the perspective of algorithms, they try to find smaller objective function values and wider distribution of the Pareto front. In Case Two, an agricultural IoT area $(240 \times 240)$ is designated. The number of sensor nodes is set to 300 and the number of agricultural IoT device nodes is set to 300 . Similarly, the number of random service requests belonging to the same type is assumed to be 30 and service facilities are all available at that moment. Compared algorithms are two different kinds of excellent algorithms: NSGA-II [56] and NNIA [20]. The maximum generations of each algorithm are set to 800 generations. Each algorithm stops until the output does not 
change for 15 consecutive generations. The setting of parameter values is given in TABLE I. Based on our pilot studies, the parameters setting of each algorithm are the most appropriate values, which are obtained through numerous experiments when each algorithm respectively achieves the optimal performance. Hypervolume is adopted in the pilot studies for setting the parameters.

TABLE I. PARAMETERS SETTING

\begin{tabular}{llllll}
\hline & & & \multicolumn{3}{c}{ IE-HCMOA } \\
parameter & NSGA-II & NNIA & GRVIA & $\begin{array}{c}\text { Sub } \\
\text { population }\end{array}$ & $\begin{array}{c}\text { Top } \\
\text { population }\end{array}$ \\
\hline Population size & 300 & 300 & 300 & 100 & 100 \\
Crossover rate & 0.9 & 1 & adaptive & adaptive & adaptive \\
Mutation rate & 0.8 & 0.1 & 0.4 & $0.3,0.4 .0 .5$ & adaptive \\
\hline
\end{tabular}

\section{B. Performance Metric}

Hypervolume (HV) [57] indicator is a kind of comprehensive performance evaluation method in the EMO area. Evaluation results can reflect convergence and diversity of the solutions set. Most of the current evaluation methods must know the PF of multiobjective optimization problem in advance. However, PF is often unknown in practical problems. Nevertheless, HV is a suitable evaluation for unknown PF. In addition, it is the indicator which is Pareto-compliant [58]. In other words, if a solutions set $S$ is better than another set $S^{\prime}, \mathrm{HV}$ value of the $S$ is higher than that of $S^{\prime}$. It measures the volume of solutions that is dominated by the approximation set, which is defined as follows in Eq. (22).

$$
H V=\operatorname{volume}\left(\bigcup_{i=1}^{n_{P F}} v_{i}\right)
$$

where $n_{P F}$ is the number of nondominated vector. For individual $i$ in the NDS, $v_{i}$ is hypervolume formed by the reference point and the member $i$. In order to reflect the scope of change of $\mathrm{HV}$ value more accurately, we adopt an improved indicator, i.e. hypervolume ratio (HVR) [59] whose definition is shown in Eq. (23).

$$
H V R=\frac{H V\left(P F_{\text {known }}\right)}{H V\left(P F_{\text {true }}\right)}
$$

where $H V\left(P F_{\text {true }}\right)$ is the hypervolume formed by optimal PF. When the solutions set is close to the true PF and has a relatively uniform and extensive distribution, the corresponding $\mathrm{HV}$ value is larger. The obtained best PF of the above compared algorithms is used as an approximation of the actual PF. Selection of the reference point is determined according to the maximum values of $f_{1}$ and $f_{2}$ in all independent runs. Its value is slightly larger than the maximum values. In the calculation of $\mathrm{HV}$ of the following two cases, reference points are $(24.5,40.1)$ and $(63.5,40.1)$, respectively.

\section{Simulation Results of Case One}

We made 30 independent runs. The experiment results based on the same conditions are as shown in Fig. 6, Fig. 7, TABLE II and TABLE III, respectively.

\section{1) Comprehensive experimental results}

The experimental results of the algorithms are presented and analyzed in TABLE II.

It displays the values of the two extreme points, which are minimum total cost of service $\left(f_{1}\right)$ and minimum service time $\left(f_{2}\right)$. They characterize the accuracy of the algorithms and the interval of the solutions distribution. Meanwhile, we compare the number of $N D S$, which is a clear demonstration of the ability of the algorithms to search the feasible solutions. In order to make the comparison more clearly, we list the average, best, worst, and variance, respectively.

It can be seen that the average results obtained by IE-HCMOA are better than those of the other algorithms from TABLE II. As for minimum value of function $f_{1}$, the average value (10.5922) of IE-HCMOA is lower than the other three algorithms. As for minimum value of function $f_{2}$, all these algorithms get the same results (10.0000) except NNIA. However, in the case of the same minimum (10.0000), corresponding $f_{1}$ value of IE-HCMOA is the lowest (14.3766). On the average values, the suboptimal is GRVIA. From the "Best", it is clear that the searched solutions of IE-HCMOA are the most excellent. From the "Worst", the $\operatorname{minf} f_{1}$ obtained by GRVIA is the lowest; the $\operatorname{minf}_{2}$ obtained by IE-HCMOA is superior to those obtained by other algorithms. From the variance of column attributes $\operatorname{minf}_{1}, \operatorname{minf}_{2}$ and Num NDS, GRVIA is the best; IE-HCMOA takes the second place. That is because the ability of GRVIA to explore better solutions is limited and tends to be stable. And the explored solution space of IE-HCMOA is larger thus it is slightly inferior in terms of variance. Despite this, from the respective of variance of $\mathrm{Num}$ $N D S$, robustness of IE-HCMOA is superior to that of NNIA and NSGA-II. We can observe that the Num NDS of IE-HCMOA is the most among these algorithms, which is due to the effective evolutionary strategies in IE-HCMOA, including the endocrine-based regulation mechanism, cooperative evolutionary mechanism among multiple subpopulations and top population, and also confirms better search ability of IE-HCMOA.

\section{2) Comparison of Pareto front}

In order to visualize the data of TABLE II, we respectively choose the best result for each algorithm in 30 runs. Selection rules are as follows. In the solutions sets with optimal or suboptimal Num NDS, select the one with optimal Pareto front.

As shown in Fig. 6, from each Pareto front of the compared algorithms, it is observed that IE-HCMOA has the best

\begin{tabular}{|c|c|c|c|c|c|c|c|c|c|c|c|c|}
\hline \multirow[b]{2}{*}{$\begin{array}{l}\text { Experi- } \\
\text { ments }\end{array}$} & \multicolumn{3}{|l|}{ NSGA-II } & \multicolumn{3}{|l|}{ NNIA } & \multicolumn{3}{|l|}{ GRVIA } & \multicolumn{3}{|c|}{ IE-HCMOA } \\
\hline & $\begin{array}{l}\min f_{1} \\
\left(f_{1}, f_{2}\right)\end{array}$ & $\begin{array}{l}\min f_{2} \\
\left(f_{1}, f_{2}\right)\end{array}$ & $\begin{array}{l}\text { Num } \\
\text { NDS }\end{array}$ & $\begin{array}{l}\min f_{1} \\
\left(f_{1}, f_{2}\right)\end{array}$ & $\begin{array}{l}\min f_{2} \\
\left(f_{1}, f_{2}\right)\end{array}$ & $\begin{array}{l}\text { Num } \\
\text { NDS }\end{array}$ & $\begin{array}{l}\min f_{1} \\
\left(f_{1}, f_{2}\right)\end{array}$ & $\begin{array}{l}\min f_{2} \\
\left(f_{1}, f_{2}\right)\end{array}$ & $\begin{array}{l}\text { Num } \\
\text { NDS }\end{array}$ & $\begin{array}{l}\min f_{1} \\
\left(f_{1}, f_{2}\right)\end{array}$ & $\begin{array}{l}\min f_{2} \\
\left(f_{1}, f_{2}\right)\end{array}$ & $\begin{array}{l}\text { Num } \\
\text { NDS }\end{array}$ \\
\hline Average & $\begin{array}{l}13.2756 \\
26.2667\end{array}$ & $\begin{array}{l}18.2686 \\
10.0000\end{array}$ & 6.0 & $\begin{array}{l}13.7244, \\
23.6000\end{array}$ & $\begin{array}{l}17.0019, \\
10.1556\end{array}$ & 5.6 & $\begin{array}{l}10.6582, \\
21.3333\end{array}$ & $\begin{array}{l}14.5996 \\
10.0000\end{array}$ & 6.1 & $\begin{array}{l}\mathbf{1 0 . 5 9 2 2}, \\
22.5333\end{array}$ & $\begin{array}{l}\mathbf{1 4 . 3 7 6 6} \\
10.0000\end{array}$ & 6.3 \\
\hline Best & $\begin{array}{l}11.3055 \\
24.0000\end{array}$ & $\begin{array}{l}15.6930 \\
10.0000\end{array}$ & 8 & $\begin{array}{l}11.4779 \\
28.0000\end{array}$ & $\begin{array}{l}14.5981 \\
10.0000\end{array}$ & 8 & $\begin{array}{l}10.4520, \\
32.0000\end{array}$ & $\begin{array}{l}14.4126 \\
10.0000\end{array}$ & 7 & $\begin{array}{l}\mathbf{1 0 . 3 0 7 0}, \\
24.0000\end{array}$ & $\begin{array}{l}\mathbf{1 3 . 8 2 6 6} \\
10.0000\end{array}$ & 7 \\
\hline Worst & $\begin{array}{l}14.9314 \\
12.0000\end{array}$ & $\begin{array}{l}24.4712 \\
10.0000\end{array}$ & 3 & $\begin{array}{l}17.6410 \\
24.0000\end{array}$ & $\begin{array}{l}21.3666 \\
10.0000\end{array}$ & 4 & $\begin{array}{l}10.8893 \\
20.0000\end{array}$ & $\begin{array}{l}14.7559 \\
10.0000\end{array}$ & 6 & $\begin{array}{l}\text { 10.9808, } \\
24.0000\end{array}$ & $\begin{array}{l}\mathbf{1 4 . 6 2 1 7} \\
10.0000\end{array}$ & 6 \\
\hline
\end{tabular}

TABLE II. Comparison of the accuracy of the extreme points and number of NDS 
convergence performance, while GRVIA the second, NNIA the third and NSGA-II the last. IE-HCMOA can find the service with the minimum total cost and service time. From the above results, we conclude that the proposed algorithms can provide optimal service selection strategies in agricultural IoT systems.

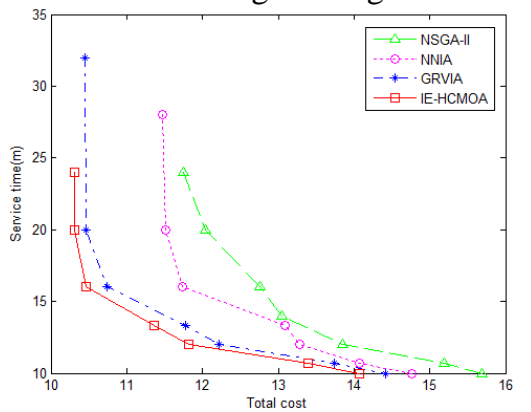

Fig. 6. Pareto front comparison of four algorithms in Case One.

3) Comparison of Hypervolume ratio

Box chart can reflect the distribution of HV ratios indicators. Fig. 7 gives the box plots for the HV ratios on the random requests described above. From minimum value, first quartile, median, third quartile and maximum value, IE-HCMOA performs remarkably better than the other compared algorithms, GRVIA, NNIA and NSGA-II. This fully demonstrates the effectiveness of the strategies proposed in this paper. From left to right, medians marked by red line are $0.7596,0.7405,0.9696$ and 0.9755 , respectively. Although the accuracy of $\operatorname{minf}_{2}$ obtained by NNIA is better than that of NSGA-II from TABLE II and Fig. 6, the median obtained by NNIA is slightly lower than that of NSGA-II in Fig. 7. The reason is that, on the diversity of solutions, NNIA is worse than NSGA-II, which also proves that the $\mathrm{HV}$ indicator is a comprehensive evaluation of the convergence and diversity.

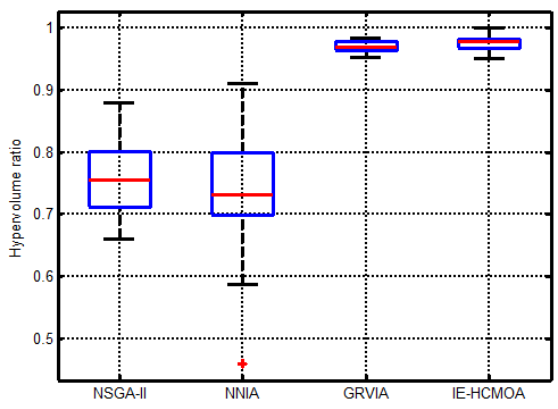

Fig. 7. Comparison of hypervolume ratio in Case One.

4) Average computing time

Average computing time is listed in TABLE III while all the algorithms are equally convergent. As indicated in the results,
NSGA-II takes the longest and average iterations are the most (343 generations). IE-HCMOA takes an average of 11.8180 seconds. After all, it employs the method of multiple populations and spends time on back and forth communication among populations. It is worthwhile to spend a little more time in exchange for lower service costs and service time if IE-HCMOA is to be applied to actual agricultural IoT.

\begin{tabular}{lcccc}
\multicolumn{5}{c}{ TABLE III. AVERAGE COMPUTING TIME } \\
\hline \multicolumn{1}{c}{ Algorithms } & NSGA-II & NNIA & GRVIA & IE-HCMOA \\
\hline Computing time (s) & $4.7160 \mathrm{e}+03$ & 18.2435 & 8.4156 & 11.8180 \\
Iterations & 343 & 226 & 76 & 96 \\
\hline
\end{tabular}

\section{Simulation Results of Case Two}

The method used in this section is identical to Case One above, but the test case is changed to 300 device nodes and 30 random requests. Therefore, the method is no longer restated in this section. 30 independent runs are made.

1) Comprehensive experimental results

The experimental results of the algorithms are presented and analyzed in TABLE IV.

From the "Average", "Best" and "Worst", $\operatorname{minf} f_{1}$ and $\operatorname{minf} f_{2}$ of IE-HCMOA are the lowest in the four algorithms. Thus IE-HCMOA achieves the best convergence performance. GRVIA ranks the second. From Num NDS, the diversity of solutions of IE-HCMOA is also superior to that of the other algorithms. From "Variance" of column attributes $\operatorname{minf}_{1}, \operatorname{minf}_{2}$ and Num NDS, variance values of $\operatorname{minf}_{2}$ and Num NDS obtained by IE-HCMOA are the lowest, which demonstrates the robustness of IE-HCMOA is also the best.

Let us contrast Num NDS index in two cases from the above results as shown in TABLE II and TABLE IV. The average of the Num NDS of IE-HCMOA is the best but variance ranks the second in Case One. The average of the Num NDS obtained by IE-HCMOA is the biggest and variance is the smallest in Case Two. Thus we conclude that IE-HCMOA is better than the other three algorithms in terms of diversity or robustness in Case Two. On the other hand, IE-HCMOA performs better on a larger scale.

\section{2) Comparison of Pareto front}

Pareto fronts of the best results for each algorithm in 30 runs are as shown in Fig. 8. IE-HCMOA ranks the first in the convergence performance. GRVIA gets the second best performance. However, solutions distribution of NSGA-II is the widest from this green curve. But when its service time reaches minimum, total cost is too large. From the point of view of practical application, too much cost in exchange for the minimum service time may not be accepted.

TABLE IV. Comparison of the accuracy of the extreme points and number of NDS

\begin{tabular}{|c|c|c|c|c|c|c|c|c|c|c|c|c|}
\hline $\begin{array}{l}\text { Experi- } \\
\text { ments }\end{array}$ & \multicolumn{3}{|l|}{ NSGA-II } & \multicolumn{3}{|l|}{ NNIA } & \multicolumn{3}{|l|}{ GRVIA } & \multicolumn{3}{|c|}{ IE-HCMOA } \\
\hline Average & $\begin{array}{l}28.0357 \\
28.4667\end{array}$ & $\begin{array}{l}39.7770 \\
11.8000\end{array}$ & 6.0 & $\begin{array}{l}30.2364 \\
26.8667\end{array}$ & $\begin{array}{l}36.8749, \\
12.4444\end{array}$ & 5.7 & $\begin{array}{l}25.1994, \\
33.6000\end{array}$ & $\begin{array}{l}30.3454, \\
12.0000\end{array}$ & 7.1 & $\begin{array}{l}\mathbf{2 4 . 8 2 4 2} \\
31.6000\end{array}$ & $\begin{array}{l}\mathbf{2 9 . 6 3 7 4} \\
12.0000\end{array}$ & 7.5 \\
\hline Worst & $\begin{array}{l}32.6676 \\
40.0000\end{array}$ & $\begin{array}{l}63.4030 \\
13.3333\end{array}$ & 3 & $\begin{array}{l}36.3866 \\
20.0000\end{array}$ & $\begin{array}{l}43.8626 \\
10.6667\end{array}$ & 3 & $\begin{array}{l}25.6553, \\
36.0000\end{array}$ & $\begin{array}{l}31.2734, \\
12.0000\end{array}$ & 5 & $\begin{array}{l}\mathbf{2 5 . 4 5 5 3}, \\
32.0000\end{array}$ & $\begin{array}{l}\text { 30.1072, } \\
12.0000\end{array}$ & 6 \\
\hline
\end{tabular}




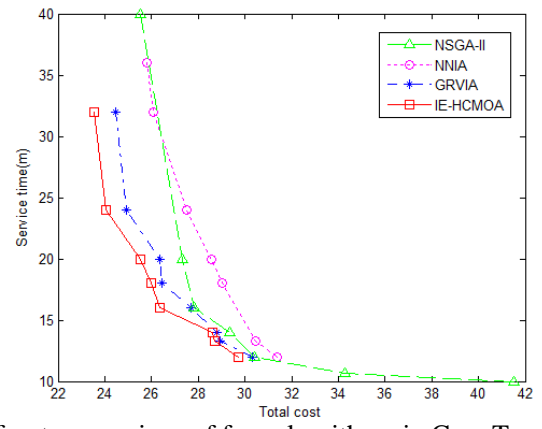

Fig. 8. Pareto front comparison of four algorithms in Case Two.

3) Comparison of Hypervolume ratio

Fig. 9 shows hypervolume ratio values of four algorithms. Obviously, IE-HCMOA performs remarkably better than GRVIA, NNIA and NSGA-II. No matter in Case One or Two, $\mathrm{HV}$ ratios of IE-HCMOA are the best, which fully demonstrates its strongest exploration ability. HV ratio of GRVIA ranks the second, which indicates that Pareto fronts found by single population are not better than those found by multiple subpopulations (IE-HCMOA) in this problem. Moreover, the fluctuation range of the solutions of NNIA is the largest and median is the lowest from Fig. 9, thus it is concluded that NNIA has the worst HVR performance in Case Two.

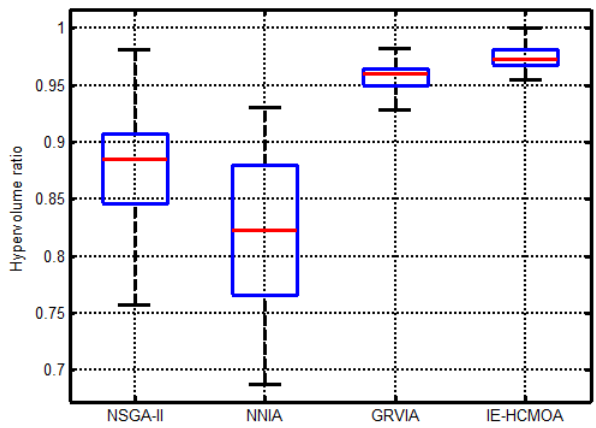

Fig. 9. Comparison of hypervolume ratio in Case Two.

4) Average computing time

Average computing time is listed in TABLE V. As can be seen from TABLE V, NSGA-II still takes the longest and average iterations are the most (482 generations). Although it runs to convergence, it is not applicable in practical applications, because the problem scenario in this paper is a real-time constrained online optimization problem. Average computing time of IE-HCMOA is 30.4422 seconds. It is perfectly acceptable in the agricultural IoT service scenarios.

\begin{tabular}{lcccc}
\multicolumn{5}{c}{ TABLE V. AVERAGE COMPUTING TIME } \\
\hline \multicolumn{1}{c}{ Algorithms } & NSGA-II & NNIA & GRVIA & IE-HCMOA \\
\hline Computing time (s) & $6.2061 \mathrm{e}+03$ & 27.0720 & 21.6189 & 30.4422 \\
Iterations & 482 & 325 & 134 & 171 \\
\hline
\end{tabular}

\section{E. Sensitivity Analysis}

To understand the influence of the parameters settings on the performance of IE-HCMOA, we conducted a set of experiments for sensitivity analysis. $w$ is varied over the set $\{0.1,0.3,0.5,0.7,0.9\}$ and $\alpha$ is varied over the set $\{0.1,0.3$, $0.5,0.7,0.9\}$. HV is used to measure the performance of each setting averaged over 100 independent runs. The experimental results are summarized in Fig. 10. For the same $w$ value, results obtained when $\alpha \geq 0.5$ are generally better than that when $\alpha=$
0.1 or $\alpha=0.3$. From Fig. 10, it can be clearly observed that results obtained when $w=0.5, \alpha=0.9$ and $w=0.7, \alpha=0.7$ are the most competitive. Finally, $w=0.7$ and $\alpha=0.7$ are adopted since the HV value resulting from this setting is slightly better.

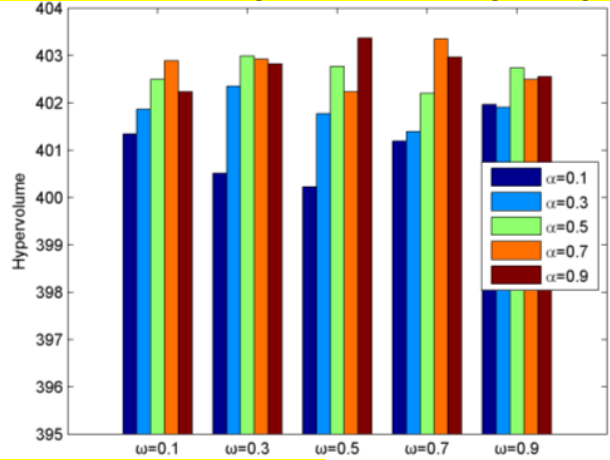

Fig. 10. Sensitivity analysis of $w$ and $\alpha$.

As for the size of subpopulation, Fig. 11 gives the box plots of the HV for different sizes (i.e., 20, 40, 60, 80, and 100) over 100 independent runs. As shown in Fig. 11, IE-HCMOA works well with a subpopulation size of 100 . Overall, the performance of IE-HCMOA improves as the subpopulation size increases.

The mutation rate used in IE-HCMOA is based on the optimal setting for GRVIA. Fig. 12 gives the box plots of the HV results obtained by GRVIA with different mutation rates. GRVIA can achieve statistically better results when the mutation rate is set to 0.4 .

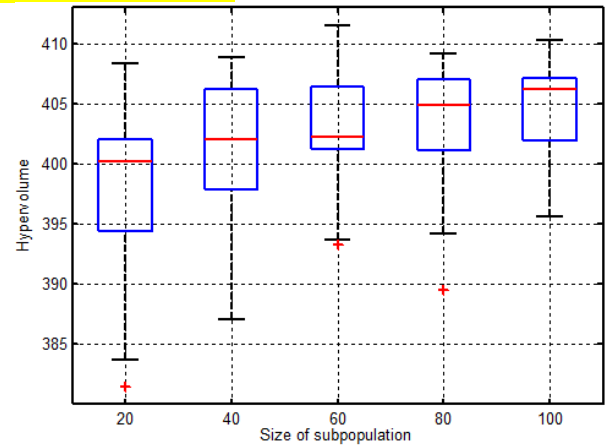

Fig. 11. Sensitivity analysis of the different size of subpopulation under the same conditions.

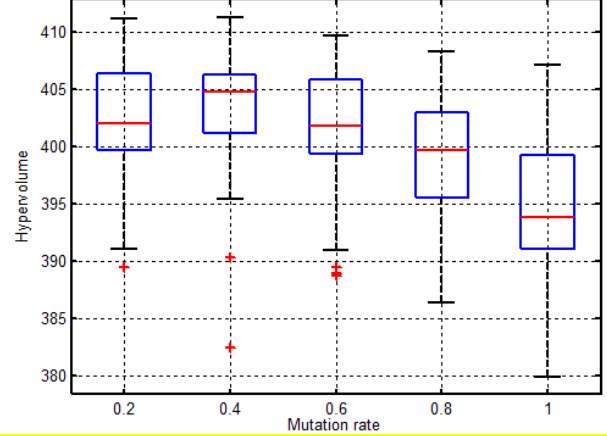

Fig. 12. Box plots of the HV results obtained by GRVIA with different mutation rates.

Note, however, that the parameters may need be further tuned if IE-HCMOA is employed to solve other problems.

\section{CONClusions}

In this paper, we build a multiobjective optimization model between service providers and multiple requests in the IoT service scenarios. In order to explore the optimal total cost and 
service time, IE-HCMOA is proposed, which uses human immune-endocrine mechanism and forgetting memory mechanism. In IE-HCMOA, GRVIA is designed as a kind method of global ranking with vaccine to choose superior antibodies, which has stronger ability of detection than NNIA and NSGA-II. Meanwhile, clustering adopted in the top population makes the operations more directional and purposeful, and it can be used to guide searching and realize self-adaptive searching. The simulation results demonstrate that the proposed algorithm can obtain the best Pareto, strong exploration ability and excellent performance.

Our future work includes developing a generic algorithm to deal with more complex dynamic situations under the environment of IoT service, and joining the transmission time to the model proposed, which will make the model more perfect. Moreover, the implementation of the algorithms on an actual agricultural IoT environment or other fields is necessary.

\section{REFERENCES}

[1] J. Branke, S. Nguyen, C. W. Pickardt, M. J. Zhang, "Automated design of production scheduling heuristics: A review," IEEE Transactions on Evolutionary Computation, vol. 20, no. 1, pp. 110-124, Feb. 2016.

[2] B. Çaliş, S. Bulkan, "A research survey: Review of AI solution strategies of job shop scheduling problem," Journal of Intelligent Manufacturing, vol. 26, no. 5, pp. 961-973, Oct. 2015.

[3] P. P. Ray, "A survey on Internet of Things architectures," Journal of King Saud University - Computer and Information Sciences, Oct. 2016. [Online]. http://dx.doi.org/10.1016/j.jksuci.2016.10.003

[4] V. Angelakis, I. Avgouleas, N. Pappas, E. Fitzgerald, D. Yuan, "Allocation of heterogeneous resources of an IoT device to flexible services," IEEE Internet of Things Journal, vol. 3, no. 5, pp. 691-700, Oct. 2016.

[5] I. Trummer, B. Faltings, W. Binder, "Multi-objective quality-driven service selection - a fully polynomial time approximation scheme," IEEE Transactions on Software Engineering, vol. 40, no. 2, pp. 167-191, Feb. 2014.

[6] Y. Chen, J. W. Huang, C. Lin, J. Hu, "A partial selection methodology for efficient QoS-aware service composition," IEEE Transactions on Services Computing, vol. 8, no. 3, pp. 384-397, May/Jun. 2015.

[7] F. Wagner, F. Ishikawa, S. Honiden, "Robust service compositions with functional and location diversity," IEEE Transactions on Services Computing, vol. 9, no. 2, pp. 277-290, Mar/Apr. 2016.

[8] Y. S. Ding, H. B. Sun, K. R. Hao, "A bio-inspired emergent system for intelligent web service composition and management," Knowledge-Based Systems, vol. 20, no. 5, pp. 457-465, Jun. 2007.

[9] Y. S. Ding, L. Gao, D. Ruan, "Communication mechanisms in ecological network-based grid middleware for service emergence," Information Sciences, vol. 177, no. 3, pp. 722-733, Feb. 2007.

[10] Y. S. Ding, Y. L. Jin, L. H. Ren, K. R. Hao, "An intelligent self-organization scheme for the Internet of Things," IEEE Computational Intelligence Magazine, vol. 8, no. 3, pp. 41-53, Aug. 2013.

[11] M. E. Khanouche, Y. Amirat, A. Chibani, M. Kerkar, A. Yachir, "Energy-centered and QoS-aware services selection for Internet of Things," IEEE Transactions on Automation Science \& Engineering, vol. 13, no. 3, pp. 1256-1269, Jul. 2016.

[12] X. N. Jin, S. J. Chun, J. Jung, K. H. Lee, “A fast and scalable approach for IoT service selection based on a physical service model," Information Systems Frontiers, vol. 19, no. 6, pp. 1357-1372, Dec. 2017.

[13] L, Li, S. C. Li, S. S. Zhao, "QoS-aware scheduling of services-oriented Internet of Things," IEEE Transactions on Industrial Informatics, vol. 10, no. 2, pp. 1497-1505, May. 2014.

[14] A. Zaslavsky, C. Perera, D. Georgakopoulos, "Sensing as a service and big data," in International Conference on Advances in Cloud Computing (ACC-2012), Bangalore, India, pp. 21-29, Jul. 2012.

[15] C. Perera, A. Zaslavsky, C. H. Liu, M. Compton, P. Christen, D. Georgakopoulos, "Sensor search techniques for sensing as a service architecture for the Internet of Things," IEEE Sensors Journal, vol. 14, no. 2, pp. 406-420, Feb. 2014.
[16] A. M. Zhou, B. Y. Qu, H. Li, S. Z. Zhao, P. N. Suganthan, Q. F. Zhang, "Multiobjective evolutionary algorithms: A survey of the state of the art," Swarm and Evolutionary Computation, vol. 1, no. 1, pp. 32-49, Mar. 2011.

[17] G. C. Luh, C. H. Chueh, W. W. Liu, "MOIA: Multi-objective immune algorithm," Engineering Optimization, vol. 35, no. 2, pp. 143-164, Apr. 2003.

[18] G. C. Luh, C. H. Chueh, "Multi-objective optimal design of truss structure with immune algorithm," Computers and Structures, vol. 82, no. 11-12, pp. 829-844, May. 2004.

[19] F. Freschi, M. Repetto, "VIS: An artificial immune network for multi-objective optimization," Engineering Optimization, vol. 38, no. 8, pp. 975-996, Dec. 2006.

[20] M. G. Gong, L. C. Jiao, H. F. Du, L. F. Bo, "Multi-objective immune algorithm with non-dominated neighbor-based selection," Evolutionary Computation, vol. 16, no. 2, pp. 225-255, Jun. 2008.

[21] L. C. Jiao, L. Wang, "A novel genetic algorithm based on immunity," IEEE Transactions on Systems, Man, and Cybernetics-Part A: Systems and Humans, vol. 30, no. 5, pp. 552-561, Sep. 2000.

[22] K. M. Woldemariam, G. G. Yen, "Vaccine-enhanced artificial immune system for multimodal function optimization," IEEE Transactions on Systems Man \& Cybernetics Part B Cybernetics, vol. 40, no. 1, pp. 218-228, Feb. 2010.

[23] R. H. Shang, L. C. Jiao, F. Liu, W. P. Ma, "A novel immune clonal algorithm for MO problems," IEEE Transactions on Evolutionary Computation, vol. 16, no. 1, pp. 35-50, Feb. 2012.

[24] N. Xu, Y. S. Ding, L. H. Ren, K. R. Hao, "Degeneration recognizing clonal selection algorithm for multimodal optimization," IEEE Transactions on Cybernetics, vol. 48, no. 3, pp. 848-861, Mar. 2018.

[25] B. H. Zhu, Y. S. Ding, K. R. Hao, "Multiclass maximum margin clustering via immune evolutionary algorithm for automatic diagnosis of electrocardiogram arrhythmias," Applied Mathematics and Computation, vol. 227 , no. 2, pp. 428-436, Jan. 2014.

[26] S. F. Adra, T. J. Dodd, I. A. Griffin, P. J. Fleming, "Convergence acceleration operator for multiobjective optimization," IEEE Transactions on Evolutionary Computation, vol. 13, no. 4, pp. 825-847, Aug. 2009.

[27] A. Lara, G. Sanchez, C. A. Coello Coello, O. Schutze, "HCS: A new local search strategy for memetic multiobjective evolutionary algorithms," IEEE Transactions on Evolutionary Computation, vol. 14, no. 1, pp. 112132, Feb. 2010.

[28] B. L. Chen, W. H. Zeng, Y. B. Lin, D. F. Zhang, "A new local search-based multiobjective optimization algorithm," IEEE Transactions on Evolutionary Computation, vol. 19, no. 1, pp. 50-73, Feb. 2015.

[29] L. Li, W. L. Wang, X. L. Xu, "Multi-objective particle swarm optimization based on global margin ranking," Information Sciences, vol. 375, pp. 30-47, Jan. 2017.

[30] J. H. Wu, J. Zhang, X. G. Zhang, Z. H. Liu, "Hierarchical co-evolution immune algorithm and its application on TSP," Acta Electronica Sinica, vol. 39, no.2, pp. 336-344, Feb. 2011

[31] Z. Y. Yang, K. Tang, X. Yao, "Large scale evolutionary optimization using cooperative co-evolution," Information Sciences, vol. 178, no. 15, pp. 2985-2999, Aug. 2008.

[32] K. C. Tan, Y. J. Yang, C. K. Goh, "A distributed cooperative co-evolutionary algorithm for multi-objective optimization," IEEE Transactions on Evolutionary Computation, vol. 10, no. 5, pp. 527 -549, Oct. 2006.

[33] C. K. Goh, K. C. Tan, "A competitive-cooperative co-evolutionary paradigm for dynamic multi-objective optimization," IEEE Transactions on Evolutionary Computation, vol. 13, no. 1, pp. 103 -127, Feb. 2009.

[34] Z. H. Zhan, J. J. Li, J. N. Cao, J. Zhang, H. S. Chung, Y. H. Shi, "Multiple populations for multiple objectives: A coevolutionary technique for solving multiobjective optimization problems," IEEE Transactions on Cybernetics, vol. 43, no. 2, pp. 445-463, Apr. 2013.

[35] J. H. Wang, W. W. Zhang, J. Zhang, "Cooperative differential evolution with multiple populations for multiobjective optimization," IEEE Transactions on Cybernetics, vol. 46, no. 12, pp. 2848-2861, Dec. 2016.

[36] S. Q. Qian, Y. Q. Ye, B. Jiang, J. H. Wang, "Constrained multiobjective optimization algorithm based on immune system model," IEEE Transactions on Cybernetics, vol. 46, no. 9, Sep. 2016.

[37] Q. Z. Lin, J. Y. Chen, Z. H. Zhan, W. N. Chen, C. A. Coello Coello, Y. L. Yin, etc., "A hybrid evolutionary immune algorithm for multiobjective optimization problems," IEEE Transactions on Evolutionary Computation, vol. 20, no. 5, pp. 711-729, Oct, 2016. 
[38] Z. H. Hu, Y. S. Ding, Q. Shao, "Immune co-evolutionary algorithm based partition balancing optimization for tobacco distribution system," Expert Systems with Applications, vol. 36, no. 3, pp. 5248-5255, Apr. 2009.

[39] Y. S. Ding, Z. H. Hu, W. B. Zhang, "Multi-criteria decision making approach based on immune co-evolutionary algorithm with application to garment matching problem," Expert Systems with Applications, vol. 38, no. 8, pp. 10377-10383, Aug. 2011.

[40] R. H. Shang, K. Y. Dai, L. C. Jiao, R. Stolkin, "Improved memetic algorithm based on route distance grouping for multi-objective large scale capacitated arc routing problems," IEEE Transactions on Cybernetics, vol. 46, no. 4, pp. 1000-1013, Apr. 2016.

[41] R. H. Shang, Y. Y. Wang, J. Wang, L. C. Jiao, S. Wang, L. P. Qi, “A multi-population cooperative coevolutionary algorithm for multi-objective capacitated arc routing problem," Information Sciences, vol. 277, pp. 609-642, Sep. 2014.

[42] R. H. Shang, B. Q. Du, H. N. Ma, L. C. Jiao, Y. Xue, R. Stolkin, "Immune clonal algorithm based on directed evolution for multi-objective capacitated arc routing problem," Applied soft computing, vol. 49, pp. 748-758, Dec. 2016.

[43] J. Tian, M. Q. Li, F. Z. Chen, N. Feng, "Learning subspace-based RBFNN using coevolutionary algorithm for complex classification tasks," IEEE Transactions on Neural Networks \& Learning Systems, vol. 27, no. 1, pp. 47-61, Jan. 2016.

[44] C. H. Mu, L. C. Jiao, Y. Liu, Y. Y. Li, "Multiobjective nondominated neighbor coevolutionary algorithm with elite population," Soft Computing, vol. 19, no. 5, pp. 1329-1349, May. 2015.

[45] W. A. Guo, M. Chen, L. Wang, Q. D. Wu, "Hyper multi-objective evolutionary algorithm for multi-objective optimization problems," Soft Computing, vol. 21, no. 20, pp. 5883-5891, Oct. 2017.

[46] X. P. Wang, L. X. Tang, "An adaptive multi-population differential evolution algorithm for continuous multi-objective optimization," Information Sciences, vol. 348, no. 2, pp. 124-141, Feb. 2016.

[47] K. Li, S. Kwong, Q. F. Zhang, K. Deb, "Interrelationship-based selection for decomposition multiobjective optimization," IEEE Transactions on Cybernetics, vol. 45, no. 10, pp. 2076-2088, Oct. 2015.

[48] Z. K. Wang, Q. F. Zhang, A. M. Zhou, M. G. Gong, L. C. Jiao, "Adaptive replacement strategies for MOEA/D," IEEE Transactions on Cybernetics, vol. 46, no. 2, pp. 474-486, Feb. 2016.

[49] C. Sauze, M. Neal, "Artificial endocrine controller for power management in robotic systems," IEEE Transactions on Neural Networks \& Learning Systems, vol. 24, no. 12, pp. 1973-1985, Dec. 2013.

[50] G. S. Yao, Y. S. Ding, Y. Jin, K. R. Hao, "Endocrine-based coevolutionary multi-swarm for multi-objective workflow scheduling in a cloud system," Soft Computing, vol. 21, no. 15, pp. 4309-4322, Aug. 2017.

[51] H. O. Besedovsky, A. Del Rey, "Immune-neuro-endocrine interactions: Facts and hypotheses," Endocrine Reviews, vol. 17, no. 1, pp. 64-102, Feb. 1996.

[52] H. Ebbinghaus, "Memory: A contribution to experimental psychology", Annals of neurosciences, vol. 20, no. 4, pp. 155-156, Oct. 2013. (Original work published 1885, Translated by H. A. Ruger \& C. E. Bussenius 1913, Reprinted with permission)

[53] S. T. Freedman, J. A. Adams, "Filtering data based on human-inspired forgetting," IEEE Transactions on Systems Man \& Cybernetics Part B Cybernetics, vol. 41, no. 6, pp. 1544-1555, Dec. 2011.

[54] W. W. Wang, B. Subagdja, A. H. Tan, J. A. Starzyk, "Neural modeling of episodic memory: encoding, retrieval, and forgetting," IEEE Transactions on Neural Networks \& Learning Systems, vol. 23, no. 10, pp. 1574-1586, Oct. 2012.

[55] V. A. Nguyen, J. A. Starzyk, W. B. Goh, D. Jachyra, "Neural network structure for spatio-temporal long-term memory," IEEE Transactions on Neural Networks \& Learning Systems, vol. 23, no. 6, pp. 971-983, Jun. 2012.

[56] K. Deb, A. Pratap, S. Agarwal, T. Meyarivan, "A fast and elitist multi-objective genetic algorithm: NSGA-II," IEEE Transactions on Evolutionary Computation, vol. 6, no. 2, pp. 182-197, Aug. 2002.

[57] E. Zitzler, L. Thiele, "Multiobjective evolutionary algorithms: A comparative case study and the strength Pareto approach," IEEE Transactions on Evolutionary Computation, vol. 3, no. 4, pp. 257-271, Nov. 1999.
[58] E. Zitzler, L. Thiele, M. Laumanns, C. M. Foneseca, V. Da Fonseca, "Performance assessment of multiobjective optimizers: An analysis and review," IEEE Transactions on Evolutionary Computation, vol. 7, no. 2, pp. 117-132, Apr. 2003.

[59] D. A. Van Veldhuizen, G. B. Lamont, "On measuring multiobjective evolutionary algorithm performance," in Congress on Evolutionary Computation (CEC2000), La Jolla, CA, US, vol. 1, pp. 204-211, Jul. 2000.

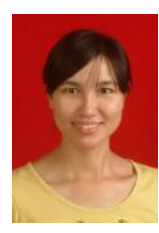

Zhen Yang is currently pursuing the Ph.D. degree at College of Information Sciences and Technology, Donghua University, Shanghai, China. She received the M.S. degree in Faculty of Information Engineering from China University of Geosciences, Wuhan, China in 2007. Her research interests include computational intelligence, evolutionary optimization, and intelligent Internet of Things.

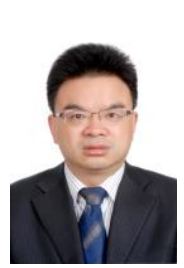

Yongsheng Ding (M'00-SM'05) was a Professor at College of Information Sciences and Technology, Donghua University, Shanghai, China. He obtained the B.S. and Ph.D. degrees in Electrical Engineering from Donghua University, Shanghai, China in 1989 and 1998, respectively. From 1996 to 1998, he was a Visiting Scientist at Biomedical Engineering Center, The University of Texas Medical Branch, TX, USA. From February 2005 to April 2005, he was a Visiting Professor at Department of Electrical and Computer Engineering, Wayne State University, MI, USA. From September 2007 to February 2008, he was a Visiting Professor at Harvard Medical School, Harvard University, MA, USA. He served as Senior Member of Institute of Electrical and Electronics Engineers (IEEE). He had published more than 300 technical papers, and 10 research monograph/advanced textbooks. His scientific interests included computational intelligence, network intelligence, intelligent Internet of Things, big data intelligence, and intelligent robots.

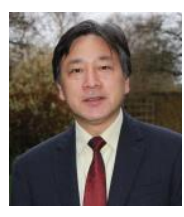

Yaochu Jin (M'98-SM'02-F'16) received the B.Sc., M.Sc., and Ph.D. degrees from Zhejiang University, Hangzhou, China, in 1988, 1991, and 1996, respectively, and the Dr.-Ing. degree from Ruhr University Bochum, Bochum, Germany, in 2001. He is a Professor of Computational Intelligence with the Department of Computing, University of Surrey, Guildford, U.K., where he is the Head of the Nature Inspired Computing and Engineering Group. He is also a Finland Distinguished Professor funded by the Finnish Agency for Innovation (Tekes), Helsinki, Finland, and a Changjiang Distinguished Visiting Professor appointed by the Ministry of Education, Beijing, China. He has co-authored over 200 peer-reviewed journal and conference papers and has been granted eight patents on evolutionary optimization. He has delivered 20 invited keynote speeches at international conferences. His research interests include computational intelligence, computational neuroscience, computational systems biology, nature-inspired, and real-world-driven problem-solving. $\mathrm{He}$ is the Editor-in-Chief of IEEE Transactions on cognitive and developmental systems and Complex \& Intelligent systems. He is also an Associate Editor or an Editorial Board Member of IEEE Transactions on Evolutionary Computation, IEEE Transactions on Cybernetics, IEEE Transactions on Nanobioscience etc.

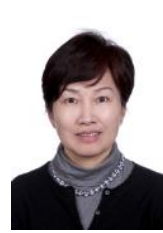

Dr. Kuangrong Hao is currently a Professor at the College of Information Sciences and Technology, Donghua University, Shanghai, China. She obtained her B.S. degree in Mechanical Engineering from Hebei University of Technology, Tianjin, China in 1984, her M.S. degree from Ecole Normale Supérieur de Cachan, Paris, France in 1991, and her Ph.D. degree in Mathematics and Computer Science from Ecole Nationale des Ponts et Chaussées, Paris, France in 1995. She has published more than 100 technical papers, and three research monographs. Her scientific interests include machine vision, image processing, robot control, intelligent control, and digitized textile technology. 